\title{
Un "Discours politique et militaire" di François de la Noue tradotto da Girolamo Naselli.L'Europa cristiana contro i Turchi
}

\section{Riccardo Benedettini}

\author{
(2) OpenEdition \\ Journals \\ Edizione digitale \\ URL: http://journals.openedition.org/studifrancesi/9098 \\ DOI: 10.4000/studifrancesi.9098 \\ ISSN: 2421-5856

\section{Editore} \\ Rosenberg \& Sellier
}

\section{Edizione cartacea}

Data di pubblicazione: 1 juin 2008

Paginazione: 101-113

ISSN: 0039-2944

\section{Notizia bibliografica digitale}

Riccardo Benedettini, «Un "Discours politique et militaire" di François de la Noue tradotto da Girolamo Naselli.L'Europa cristiana contro i Turchi», Studi Francesi [Online], 154 (LII | I) | 2008, online dal 30 novembre 2015, consultato il 12 janvier 2021. URL: http://journals.openedition.org/studifrancesi/9098 ; DOI: https://doi.org/10.4000/studifrancesi.9098

\section{(c)}

Studi Francesi è distribuita con Licenza Creative Commons Attribuzione - Non commerciale - Non opere derivate 4.0 Internazionale. 


\section{Un "Discours politique et militaire" di Francois de la Noue tradotto da Girolamo Naselli. L'Europa cristiana contro i Turchi}

Girolamo Naselli partecipò intensamente alla vita editoriale e culturale della città di Ferrara. Per i tipi di Benedetto Mammarello, nel giugno 1590 lo scrupoloso e fedele traduttore pubblicò Dell'origine, conservatione, et decadenza degli Stati di René de Lucinge e, nel novembre dello stesso anno, il suo nome si legò a quello della prima versione italiana degli Essais di Montaigne ${ }^{1}$. Della sua traduzione del Modo di vincere $i$ Turchi, E scacciarli d'Europa con la Lega dei Prencipi Christiani, due copie identiche sono conservate alla Biblioteca Nazionale Centrale di Firenze (Magliabechiano 4.4.303/a e Magliabechiano 4.4.303/c) 2. I duplici esemplari a stampa di questo ventiduesimo Discours dell'illustre capitano ugonotto François de la Noue (i cui Discours politiques et militaires sono pubblicati a Basilea nel 1587 dall'editore Philippe Canaye de Fresne presso la stamperia François Forest ${ }^{3}$ ) sono seguiti dal Parere come si pos-

(1) Al riguardo, come per ogni indicazione di ordine bio-bibliografico concernente il traduttore estense, si veda: A. M. RAUGEI, L'onesta infedele: ancora sulla traduzione degli "Essais" di Girolamo Naselli, in Montaigne e l'Italia, «Gruppo di Studio sul Cinquecento francese. 2», Atti del Congresso Internazionale di Studi di Milano-Lecco, 26-30 ottobre 1988, Genève, Slatkine, Moncalieri, Centro Universitario di ricerche sul viaggio in Italia, 1991 , pp. 35-66; di chi scrive, Il "De la Naissance, duree, et cheute des estats" da René de Lucinge a Girolamo Naselli. Osservazioni sulle modalità di traduzione Pubblicazioni dell'Università di Bari, «Annali della Facoltà di Lingue e Letterature Straniere», terza serie / 2006-2007 / XVIII, Schena editore, pp. 409. 423. Riconosciamo qui il debito che hanno entrambi i nostri studi nei confronti del lavoro di Anna Maria Raugei.

(2) DEL MODO DI VINCERE I TURCHI, / E scacciarli d'Europa / CON LA LEGA DEI / PRENCIPI CHRISTIANI. / DISCORSO DEL SIG DELLA NOUE . / Tradotto da Girolamo Naselli Ferrarese / dalla lingua Francese nell'Italiana. / (marca editoriale) / In FERRARA, Appresso Vittorio Baldini, / Stampatore Camerale M.D.C. La marca editoriale sul frontespizio è attribuita ad Anselmo Giaccarelli. Le citazioni sono fatte dalla prima copia. Nella numerazione delle pagine del testo $\left(4+128\right.$, in $\left.-8^{\circ}\right)$ sono presenti due errori: le indicazioni, sempre in alto a destra, delle pp. 2425 sono ripetute due volte, per cui non vengono segnalate le pp. 26-27. Nella trascrizione a seguire del testo si è introdotta l'opposizione tra i grafemi $i / j$ e $u / v$, si sono divise (o unite) le parole, si è seguito l'uso moderno per accenti, apostrofi e distribuzione di maiuscole; si sono sciolte le \& e le $\beta$; si è conservata invece l'interpunzione cinquecentesca, perché essa ci consente un più rigoroso riscontro con l'edizione francese. Abbreviamo d'ora in poi il titolo della resa italiana in Discorso e l'originale in Discours, mettendo tra parentesi nel testo, subito dopo la citazione, il numero della pagina cui rimandiamo preceduto, nel caso del Discorso, dal numero di capitolo voluto da Naselli.

(3) Nostro testo base di riferimento per il raffronto linguistico con la traduzione italiana è Fr. DE La Noue, Discours politiques et militaires, Publiés avec une introduction et des notes par F. E. SuTCLIFFE, Genève, Droz («Textes littéraires français»), 1967, edizione critica cui si rinvia per la storia editoriale, le fonti e la fortunata diffusione dell'opera. Abbiamo consultato inoltre i due esemplari a stampa conservati presso la Biblioteca Nazionale Centrale di Firenze: DISCOURS / POLITIQUES / ET MILITAIRES / DU SIEUR DE / LA NOUE. $/ * * *$ / Recueillis \& mis en lumiere par le Sieur DE / FRESNES \& dediez au Roy tres-Chrestien / HENRY IIII de ce Nom. / Derniere Edition enrichie de deux Indices, / dont le premier est des Sommaires \& ar / gumens sur chasque Discours Le Second, / des choses plus notables contenues en / tout l'œuvre. / (marca editoriale) / A LYON, / Pour DANIEL BELLON. / 1595 (Magliabechiano $20.7 .149 ; 16+1019+36$, in-16 $\left.{ }^{\circ}\right) ;$ DISCOURS 
sa resistere a'Turchi, di Lazarus von Schwendi, testo anch'esso tradotto da Naselli e pubblicato sempre nel 1600 a Ferrara per conto della Stamperia Camerale di Vittorio Baldini ${ }^{4}$.

Dal confronto fra le tre traduzioni, emergono alcuni tratti caratteristici. Pur cercando di fornire un testo il più possibile vicino all'originale, Naselli interviene per una più agevole fruizione. In questo senso si interpretano un certo numero di scarti di ordine essenzialmente tecnico-linguistico: riduzione delle figure retoriche, semplificazioni a livello semantico, incidenti paleografici nella lettura dei numeri, aumento di chiarezza in ambito verbale. Ma ancora più importanti sono le differenze, derivanti dalle diverse pretese ideologico-letterarie che i tre testi avanzano. Gli elogi alla Cattolicissima Spagna e le condanne alla religione riformata che il membro della Lega Lucinge lascia nel proprio trattato, sono evidentemente conformi alla politica di Alfonso II. Il traduttore cerca di non modificarne le idee-guida, e i «problemi» di traduzione provengono soprattutto da ambiguità terminologiche. Nel caso invece del Discorso di La Noue, l'officina di Vittorio Baldini dovette riconoscere evidenti pericoli di censura legati al protestantesimo dell'autore . Come già era avvenuto per la controversa opera di Montaigne, Naselli tende, da un lato, ad attenuare le parti più compromettenti e meno utili alla riflessione morale, politica e militare, dall'altro a sopprimere quelle sezioni il cui contenuto, attuale ai tempi della stesura da parte di La Noue, non suscita più interesse per il lettore italiano. Si considerino le tardive risposte su come evitare europee lotte intestine, oramai risoltesi da tempo. Un'ulteriore esclusione riguarda quei punti dove più si avverte l'individualismo dell'autore, intervento che si presenta già nelle prime frasi del testo. Per essere edito, il Discorso, dallo stile elegante ma anche qua e là arricchito di artistici tocchi di colore, deve rispondere alle richieste di un preciso programma editoriale, ben attento ad immettere sul mercato un testo non compromettente, non suscettibile di provocare scontento nel pubblico di lettori e, soprattutto, di acquirenti.

Tenendo conto di queste esigenze, si possono interpretare molteplici modifiche al testo originale, a partire dalla scelta di un diverso titolo sul frontespizio dell'edizione italiana. Se leggiamo il Discours nel contesto del libro in cui è inserito comprendiamo bene la densa compattezza della sua struttura. Dei Discours politiques et militaires $^{6}$, il ventiduesimo rientra nei «Quattro Paradossi Militari», segnatamente nel

/ POLITIQUES ET / Militaires du Seigneur / de la Nouë. / NOUVELLEMENT RE / cueillis et mis en lumiere. / (marca editoriale) / A BASLE, / De l'Imprimerie de François Forest / M.D.XCVII. (Magliabechiano 4.1.216; $8+711$, in $-4^{\circ}$ ).

(4) Sul principale editore ferrarese del secolo, vedi R. GORRIS, "Prudentia perpetuat": Vittorio Baldini, editore ferrarese di Francesco Patrizi, in Francesco Patrizi filosofo platonico nel crepuscolo del Rinascimento, a cura di P. CASTELLI («Pubblicazioni dell'Università di Ferrara», VIII), Firenze, Leo S. Olschki editore, 2002, pp. 219-252.

(5) Ricordiamo che le memorie di La Noue sono precedute da una lettera dedicatoria (1-8), scritta a Losanna il primo aprile 1587, nella quale Canaye de Fresnes si rivolge al re di Navarra. L'editore, ancor giovane giurista, è passato quindicenne al calvinismo (ritornerà al cattolicesimo durante la monarchia restaurata del primo dei Borboni) ed è sempre rimasto affascinato dall'ideale dell'unità cristiana. Rielaboratore dell'Organon aristotelico, in cui scorge soprattutto uno strumento esemplare di quell'arte retorica «qui donne les moyens d'examiner toutes doctrines», e quindi di risolvere anche ogni controversia religiosa, de Fresne riconosce nell'opera di La Noue un invito alla «concordia politica» e alla pacifica convivenza dei seguaci cattolici e protestanti. A seguito di un viaggio in Turchia nel 1572, de Fresne scrive una relazione positiva del modo in cui i turchi amministrano la giustizia (il Corano, il libro-codice divino, è anche un testo giuridico e il profeta Maometto è un politico legislatore), voyage noto a La Noue (sull'opinione dell'autore circa i viaggi di formazione si legga il Discours $V$, p. 143 e sg.). Sul Viaggio di de Fresne, vedi A. BETTONI, Le Isole dell'A Archipelago' nel "Voyage" di Philippe du Fresne-Canaye, in L'Europa e il Levante nel Cinquecento. II. Le Isole del Mediterraneo orientale nella letteratura di viaggio, a cura di L. ZILLI, Padova, Unipress ( «Gruppo di Studio sull'Europa e il Levante»), 2004, pp. 149-176.

(6) Il testo è organizzato in ventisei Discorsi: «Discours» (I-XVII, pp. 17-306), «QUATRE PARADOXES MILITAIIRES» (XVIII-XXV, pp. 
quarto (382-456), ed è preceduto da «QUE LES ALLIANCES FAI / tes par les Princes Chrestiens avec les Mahumetistes, enne / mis capitaux du nom de Christ, leur ont toujours esté mal / heureuses, \& qu'on ne se doit point allier estroittement avec / ques eux. / VINGTUNIEME DISCOURS», nel quale La Noue vuol raccontare a grandi linee l'«origine de la nation turcque» (418), nel suo ingrandimento dell'impero che mette in pericolo «les Estats de la Chrestienté, depuis le plus grand jusques au plus petit» (419). Raccomandando l'«utilité de tous ces discours turquesques» (429), l'autore si domanda «en quelle manière un prince chrestien peut s'allier \& avoir estroite communication avec tels barbares, qui sont comme marquez \& destinez pour estre les flagellateurs des chrestiens?»(431-432). Le parole conclusive del Discours, «Car il ne faut pas seulement se desallier d'avec eux, mais aussi il faut haïr leur malice, $\&$ tascher par la force \& toutes autres voyes honnorables de les renger à ce poinct qu'ils ne puissent endommager la Chrestienté», ne esprimono concisamente l'idea di fondo. Quanto più si ritiene non esserci alcuna salvezza al di fuori del Cristianesimo, tanto più il legame tra politica e religione si orienta verso quest'ultima. Si deduce così che se l'alleanza col Turco non ha ragione di esistere, la guerra contro «ces barbares, qui sont les instrumens de l'ire de Dieu» (429) darà invece al mondo cristiano una maggiore coscienza della propria unica entità morale ${ }^{7}$. Naselli, certo in stretta collaborazione con l'editore, organizza invece il materiale unico del discorso in 58 capitoli, manipolazione che, pur nel rispetto della linea compositiva originale, ne frantuma, almeno formalmente, la solida unità.

I titoli assegnati sono elencati nell' «INDICE DE' CAPI, / Che si contengono nell'Opera» $(2 \mathrm{r}-4 \mathrm{v})$ e ripresi ad ogni inizio di capitolo. In prima pagina, al nuovo «DISCORSO / DEL SIGNOR DELLE NOUE, / Del modo di debellare i Turchi» segue il titolo «CHE LI PRENCIPI CHRISTIANI / ben'uniti, possono in quattro / anni scacciare i Turchi d'Europa», 'onesta' traduzione dell'originale, «QUE LES PRINCES CHRESTIENS estans bien unis ensemble peuvent en quatre ans chasser les Turcs de l'Europe». Lo scrupolo nel sezionare il materiale - risultato del lavoro volto a riconoscere il carattere individuale e stabile del singolo Discorso, di fatto considerato indipendente dall'opera originale nella sua interezza - arriva al punto di ritoccare o interrompere il flusso delle immagini, concrete e dinamiche, che La Noue riporta a conferma dimostrativa della propria esposizione. Il capitolo VI, «Violenze horribili de'Turchi» (7-8), strettamente connesso al precedente, «Modo di reprimere l'insolenza de'Turchi» (8-9), relativo alla necessità della guerra, acquista una propria autonomia, probabilmente per fermare l'attenzione su due gravi e scioccanti violenze commesse dai turchi contro i cristiani: i sorprendenti rapimenti di bambini, per convertirli alla religione musulmana («la fausse doctrine de Mahumet», 428), e quelli degli adulti, da utilizzarsi come schiavi ${ }^{8}$. Lo shock è già, nel tardo Cinquecento, un importante criterio di valore e incentivo al consumo. E ulteriore conferma del nuovo impianto narrativo è data quando, trattando della necessità di sciogliere la lega con Solimano il Magnifico, contratta nel 1535 dal Cristianissimo («Come gli altri Prencipi

307-540) e «OBSERVATIONS SUR PLUSIEURS CHOSES ADVENUES AUX trois premiers Troubles, avecque la vraye déclaration de la pluspart d'icelles» (XXVI, pp. 544-710).

(7) La posizione di La Noue, è noto, sembra costruirsi su un insanabile dualismo. Se qui l'autore sostiene che «le mot de prochain s'estend indifferemment à tous hommes» (92), nel Discours XXII egli propone invece lo sterminio degli infedeli, azione necessaria per sconfiggere il male e affermare nel mondo il verbo del «vero IDDIO» (LVI, 123), idea senza dubbio in contrasto con la religiosità intesa come sentimento di Dio, immanente nell'uomo, tale che in esso tutti gli uomini sono uguali e comunicano.

(8) Su «le sort des captifs», come sull'immagine del Turco nell'Europa moderna, vedi G. TurBeTDelof, L'Afrique Barbaresque dans la Littérature Française aux XVI et XVII siècles, Genève, Droz, 1973. 
potrebbono essere indotti a congiungersi con li tre sudetti», XI, 17-18), Naselli isola nelle «Ragioni per la Lega di Francia col Turco» (XII, 19-21) l'esempio di Francesco I: mosso da un'apprensione strategica - il timore di subire attacchi da parte della Spagna, della Germania, dei Paesi Bassi, dell'Inghilterra e dell'Italia - il re di Francia dà vita all'allenza franco-turca. All'origine di questi rimaneggiamenti sta la volontà di richiamare l'attenzione su un blocco omogeneo di discorso all'interno di un sistema basato invece sulla più estesa fluidità dello scritto memoriale. L'opera di testimonianza richiede l'espressione del punto di vista di colui che scrive, qualità che potrebbe minare la garanzia di 'corretta' conformità che lo Stampatore ferrarese sembra esigere dal testo. L'incipit del Discorso presenta un esempio tipico di omissione della soggettività:

«IL seroit mieux seant à quelques capitaines, que j'estime qui vivent encores aujourd'buy, comme le seigneur Jean André Doria Italien, Lazare Schvende Alemand, E le chevalier de Rommegas François, qui se sont trouvez en plusieurs guerres contre les Turcs, de discourir des moiens pour reprimer leur puissance, qu'à moy qui n'ay jamais veu bransler leurs estandars par mer ny par terre, ny recogneu leurs frontieres» $(437) \rightarrow$ «CONVERREBBESI molto più ad alcuni eccellenti Capitani, i quali si sono trovati in molte guerre contra i Turchi, l'havere discorso de'modi per reprimere la loro possanza, che a me, che poco ho veduto movere i loro stendardi per mare, et per terra, né riconosciute le loro frontiere» (I, 1).

Già in questo passo si avverte la necessità di attenuare l'opinione privata di La Noue, anche se l'esclusione del «j'estime» (ancor più significativa se teniamo conto della posizione iniziale da esso rivestita) comporta la soppressione degli esempi, persino i nomi del principe Andrea Doria, e quello del barone Lazarus von Schwendi, il cui Parere segue qui il Discorso. La stessa soluzione è raggiunta in altri due casi:

«les ministres de nostre Roy» (449) $\rightarrow$ «Ministri del Re Christianissimo» (XIII, 21);

«Sa Majesté Catholique, qui est doüee (ce dit-on) de grand debonnaireté, et en fait journellement des preuves en plusieurs fors qu'en moy» (452) $\rightarrow$ «La Maestà Catholica, che è dolce, et di grande benignità» (XVI, 26),

testimonianze nelle quali la traduzione rende il testo molto più oggettivo, concretamente indifferente alla presenza «d'une forte personnalité» che, sostiene Sutcliffe, «nous donne l'impression d'écouter une voix, de nous laisser conduire par un guide qui s'adresse directement à chacun de nous»?

La precisa cautela del programma editoriale giustifica ed orienta alcune esclusioni compiute dal traduttore quando l'argomento del Discorso fa riferimento alle autorità più importanti, il Papato e l'Impero. Se la religione è in grado di tenere uniti gli uomini («l'union chrestienne que devons tous desirer», Discours $I V, 125$ ), non è la diversità delle religioni a dover spingere i francesi a «s'estimer comme Turcs les uns les autres» (Discours I, 20). Il Turco, menzionato qui per la prima volta, non lascia ancora trasparire quel senso di minaccia che risulterà di lì a poco nel Discours XXII ${ }^{10}$. Ed infatti La Noue continua osservando: «Car puis que chacun confesse qu'il adore

(9) «Introduction», cit., p. XXXII.

(10) La Noue osserva che i turchi, per timore della punizione divina, «s'abstiennent du blaspheme» (24). La virtù dei mussulmani, definiti comunque «des pauvres aveugles», li rende, almeno da questo punto di vista, più rispettosi dei francesi, tra i quali, continua La Noue, il detestabile vizio di abusare del nome di Dio è frequente persino tra i ragazzini di sette, otto anni e tra i contadini, vale a dire tra coloro che vivono lontani dalle corti (la corte e $\mathrm{Pa}$ rigi sono le due luci che devono illuminare tutta la Francia, si legge oltre, p. 52). 
un mesme Dieu, advouë pour Sauveur un mesme Jesus Christ, \& que les Escritures $\&$ fondemens sont semblables, il doit y avoir telle fraternité \& charité entre eux, que cessans toutes haines, cruautez \& guerres, on vienne à quelque reconciliation». Non è alla religione che si deve imputare di «apporter tant de maux» in Europa, quanto alla «malice des hommes, qui aiment mieux les tenebres que la lumière, \& à l'ignorance d'iceux, en ce qu'il leur semble que telles contrarietez se doivent resoudre par le fer \& par le feu, au lieu qu'elles se devroyent composer par la voye de douceur» (Discours II, 72). Tuttavia questo atteggiamento di sereno confronto fra le fedi - nel quale si lasciano intravedere anche aspirazioni e speranze ireniche, quegli stessi ideali che avevano spinto il giovane Canaye de Fresne a pubblicare l'opera, rivelatasi subito di ampia fortuna ${ }^{11}$-, manca problematicamente nel Discorso ferrarese, dove La Noue si lascia andare ad affermazioni di propaganda estrema, cattolica o protestante che sia, volta a:

«empescher les horribles degasts que ceste cruelle nation continue sur leurs sujets» (444) $\rightarrow$ «vietare gli horribili guasti, et distruttioni di questa crudele natione, continuate su i loro sudditi» (IX, 13-14).

Ma se qui Naselli ricorre all'utilizzo di sinonimi glossanti per rendere il termine «degasts» ${ }^{12}$, per denunciare con forza la gravità degli inequivocabili rischi di un'espansione turca, altrove l'omissione gli deve apparire come il solo mezzo per conservare la conformità del testo alle esigenze dell'ambiente intellettuale ferrarese. In questo stesso capitolo IX, leggiamo che, sotto la guida di Dio, l'imperatore, i re, i principi e le varie repubbliche devono difendere i propri popoli, come quei pastori che hanno sempre gli occhi aperti per guardare che i lupi non assaltino le loro greggi ${ }^{13}$. La strada migliore per riuscire in questo intento consiste, spiega La Noue con progressione («Chi sono quelli, che devono cominciare a porre gl'altri in camino», X), nel far sì che coloro che hanno autorità all'interno del sistema della Cristianità mostrino a tutti «les dents \& les griphes du lyon» (446; il «leone» - lo stesso che l'esperienza mostra a Machiavelli, insieme alle volpi e ai lupi - diventa in Naselli un «drago», X, 15, errore che, apparentemente inspiegabile, comporta una variata interpretazione del simbolismo legato all'animale $\left.{ }^{14}\right)$. In questo compito di convincere la collettività dei

(11) I Discours hanno tanta fortuna che dopo la prima edizione basileese del 1587 altre cinque ne seguono fino al 1590: nel 1587 vengono stampate due edizioni, una sempre a Basilea e una a Losanna; altre due sono edite nel 1588 e una quinta nel 1590 . Nel 1596 l'opera è nuovamente ristampata a Basilea da Jacob Stoer.

(12) Il ricorso ai sinonimi glossanti, modalità che sappiamo molto frequente nella traduzione del testo di Lucinge, compare altrove nel Discorso: «Puis apres cecy s'entreprint le memorable voyage de Godefroy de Bouillon» (465) $\rightarrow$ «Poi doppo questo s'attese all'impresa, et viaggio memorabile di Goffredo di Bouillone» (XXVII, 45); «plusieurs autres seigneurs» $(466) \rightarrow$ «molti altri infiniti Signori, et Baroni» (XXVII, 45); «ceste glorieuse conqueste» $(500) \rightarrow$ «questa gloriosa impresa, et acquisto» (LI, 100); «les forçaires» (506) $\rightarrow$ «forzati, et schiavi» (LIV, 111). Segnaliamo anche altri esempi di glossa: «car si aucune guerre fut onc necessaire cestecy l'est» (441) $\rightarrow$ «perché se mai guerra alcuna fu necessaria, questa è necessarissima» $(\mathrm{V}, 7)$, in cui il pronome viene esplicitato; «aussi bien que l'autre»
(449) $\rightarrow$ «così bene come il Turco» (XII, 20); parlando di Guicciardini (ricordiamo che La Noue si è dedicato ad un commentaire sulla Historia del fiorentino), «j'allegueray ses propres paroles, car elles meritent d'estre poisees» (471) $\rightarrow$ «io allegherò le proprie parole del lib. 13 della sua bistoria, perché esse meritano d'essere intese» (XXXIII, 55); «Mais quand on n'auroit pour le commencement exploité que le quart de ceci (488) $\rightarrow$ Ma quando non si fosse essequito, che la quarta parte di ciò» (XLIV, 81); «le tiers des galeres ennemies» (506) $\rightarrow$ «la terza parte delle galere nimiche» (LIV, 112); «de l'ordre et de la discipline» $(460) \rightarrow$ «dell'ordine, et della disciplina militare» (XXII, 36). E infine un esempio di rafforzamento dell'aggettivo: «certes il faut de fortes barrieres» (443) $\rightarrow$ «certo bisognano de' ripari molto forti (VII, 11); ed uno di introduzione dell'aggettivo: «qu'un pere à ses enfans» (452) $\rightarrow$ «come un buon padre a' suoi figliuoli» (XIV, 25).

(13) L'immagine del lupo e della pecora, di chiara ascendenza evangelica $(M t, 7,15-16)$, compare anche nel Discours XXI, p. 426.

(14) Sul significato simbolico del «leone» e del 
cristiani ad unirsi per portare avanti una guerra giusta quanto necessaria, nella quale ogni membro consacri le proprie energie al trionfo della gloria di Dio, sono coinvolti innanzitutto il papa, l'imperatore e il re di Spagna:

La premiere personne requise pour persuader avec efficace, seroit le pape, la dignité duquel est beaucoup reveree des princes catholiques, vers lesquels il envoyeroit solennellement. Et quand ils verroient qu'il ne crieroit plus apres eux, comme il fait à present, disant, Coupez la gorge à vos sujets qui ne me veulent pas recognoistre, ains auroit changé de langage, il est certain que ces inductions auroient beaucoup de vertu, comme elles eurent des autres papes aux premiers voyages, entrepris pour le recouvrement de la Terre saincte. La seconde personne necessaire seroit l'empereur; car encores que sa puissance ne soit maintenant conforme au titre $q u$ 'il porte, si est-ce que la sacree dignité, dont il est revestu, doit estre en grande reverence à tous les potentats chrestiens, les remonstrances duquel auroient aussi grand pouvoir envers toute la Germanie (446-447) $\rightarrow$ La prima persona per persuadere con efficacia, sarebbe il Sommo Pontefice, la dignità del quale è molto riverita da' Prencipi Catholici, verso i quali egli mandasse solennemente. Et quando vedessero, che instasse gagliardamente, cosa certa è, che queste induttioni haverebbono gran forza, com'hebbero già degl'altri Pontefici ne'primi viaggi, abbracciati per la ricuperatione di Terra Santa. / La seconda persona necessaria sarebbe l'Imperatore: perché la dignità sacra, della quale è rivestito, deve essere in grande riverenza a tutti li Potentati Christiani, le rimostranze del quale havrebbono anche forza grande verso tutta la Germania (X, 15-16).

Si tratta di due scarti evidenti. Il primo è volto a garantire la dirittura spirituale del papa, invocato come Pontefice Massimo ${ }^{15}$; il secondo esclude ogni giudizio sulla indefettibile veste imperiale. Il difficile parallelismo fra Chiesa e Stato viene risolto da Naselli con paritarie ed appiananti modalità di traduzione, che ci permettono di riconoscere la continua e complessa interazione tra la lingua e il divenire della storia. Se è vero che il protestantesimo divenne la religione ufficiale dell'Europa settentrionale, mentre il cattolicesimo rimase quella delle regioni meridionali del continente (fatta eccezione per l'Irlanda), non c'è dubbio che l'impegno della Chiesa romana e dell'Impero, ognuno nei singoli ambiti territoriali, presupponeva secondo La Noue una prevalenza del potere religioso su quello politico. Ma la prudentia perpetuat del motto baldiniano diviene per Naselli ispirazione di un cauto uso della lingua, la stessa cautela che riscontriamo quando, si legge, è opportuno che l'imperatore

«drago», vedi J. Chevalier, A. Gheerbrant, Dictionnaire des symboles. Mythes, Rêves, coutumes, gestes, formes, figures, couleurs, nombres, Paris, Seghers, 1974 [1969] $]^{1}$, ad voces. Si ricordi la presunta profezia riferita ad Enrico IV, «super aspidem et basiliscum deambulabis et conculcabis leonem et draconem», pronunciata di lì a poco da Sisto V, nella quale il leone avrebbe rappresentato la Spagna e il drago lo stesso pontefice (l'aspide il duca di Mayenne e il basilisco Carlo Emanuele di Savoia). Nel Discorso compaiono ulteriori scarti per i quali ci sembra difficile ricostruire l'alterazione che ne è causa, probabilmente una lettura erronea dell'originale o una scrittura corrotta nella resa italiana: «ce qui nous doit asseurer que leurs enfans ne seront pas invincibles» $(454) \rightarrow$ «il che ci deve assicurare, che ne anche bora non saranno invincibili» (XVII, 27 ); «les beaux artifices des chefs» (493) $\rightarrow$ «gl'artificii bellici de' Capitani» (XLVIII, 90), dove belli passa a bellici.
(15) Su questa censura operata da Naselli, vedi B. ConCONI, Chrétiens et Turcs: La comparaison des religions à la Renaissance, in L'Europa e il Levante nel Cinquecento. I. Cose turchesche, a cura di L. ZILLI, Padova, Unipress («Gruppo di Studio sull'Europa e il Levante»), 2001, pp. 113-144: p. 137 n. 93. Del resto già nel Discours XXI La Noue si era espresso in termini simili: «Ce seroit contre ceux-cy que le pape devroit tourner ses fulminations, \& plusieurs potentats chrestiens leur courroux \& leurs armes, \& non contre leurs propres sujets, ausquels ils improperent à tort le crime d'heresie. Car ce sont les Turcs qui sont les vrais heretiques. On doit tirer l'espee contr'eux, non pas pour les convertir (pource que l'Evangile ne se plante és entendemens des hommes qu'avec la predication \& la bonne vie) mais pour reprimer leur cruauté \& tyrannie; \& telles guerres seroient aussi necessaires que nos domestiques sont miserables» $(432-433)$. 
«assignast le lieu (moyennant que le pape n'en eust jalousie) où les ambassadeurs des grands princes se trouveroient» (455) $\rightarrow$ «assegnasse il luogo (pur che il Papa se ne contentasse) dove gl'Ambasciatori de'Prencipi grandi si ritroverebbono» (XVIII, 30).

L'espressione «aver gelosia», che attribuisce al papa sentimenti e aspettative troppo comuni, assume una veste più dignitosa nella resa italiana.

La stessa tendenza attenuativa motiva tre ulteriori esempi di omissione riguardanti la Chiesa e l'Impero. Avendo a cuore la ripresa della potenza francese, indebolita dalle tragiche conseguenze delle lotte religiose, La Noue non trascura il fatto che il Cristianissimo debba partecipare alla crociata contro l'Islam ${ }^{16}$. Col pretesto della difesa della fede cristiana, i principi che avessero partecipato alla lotta ne avrebbero ricavato una serie considerevole di vantaggi economici, tutti rischiosamente

«au profit de la maison d'Austriche, qui est desja montee à un si haut degré de grandeur, qu'elle fait peur à tous ses voisins» (449) $\rightarrow$ «ad utile della Casa d'Austria» (XII, 21).

La proposta di un'equa distribuzione del bottino di guerra trova conferma negli esempi del passato, e in particolare nelle considerazioni politiche su don Giovanni d'Austria, «Et quant aux deniers que leur Empereur tire de ses thresors de Constantinople en temps de guerre, il les remet en temps de paix» (463), sentenza esplicita che Naselli omette del tutto (XXV, 41). Analogamente, la compromettente equazione che La Noue stabilisce ponendo in rapporto i papi e i principi da un lato e la crociata e il contributo economico dall'altro, induce il traduttore ad abbandonare queste considerazioni:

«Ce moyen, soit qu'il vinst maintenant par les papes, ou par l'authorité des princes, et qu'on l'appellast croisade ou contribution, seroit pourtant necessaire pour aider aux potentats à satisfaire aux despenses» (456-457) $\rightarrow$ «questo modo sarebbe per tanto necessario per aiutare i Potentati a satisfare alle spese occorrenti» $(\mathrm{XX}, 32)$.

Il richiamo del sovrano di Francia alla Crociata, che testimonia molto chiaramente come il conflitto di carattere utilitario fosse travestito da conflitto ideologico, è un fattore che sembra favorire l'intervento di altri potentati europei e fare da «contrappeso» nei confronti della Spagna dominatrice e dei principi di Germania ${ }^{17}$. Nella lega contro il Turco entrerebbe «mesmement le roy qui maintenant regne en Pologne» (450), proposito invece escluso da Naselli (XIII, 23) ${ }^{18}$. Al momento della stesura del Discours, la Polonia rappresenta anche per La Noue un paese (ufficialmente cattolico) dalla complicata situazione politica: dopo la morte nel 1572 di Sigismondo II,

(16) Sull'appello a scendere in campo contro il nemico musulmano in nome dell'antico ideale di un corpus christianorum, vedi J. Balsamo, Il Turco vincibile. Un «corpus turc» à la fin du XVI siècle. La Noue, Naselli, Soranzo, Esprinchard, in Scritture dell'impegno dal Rinascimento all'età barocca, «Gruppo di Studio sul Cinquecento francese. 7», Atti del Convegno Internazionale di Studio, Gargnano, Palazzo Feltrinelli, 11-13 ottobre 1994, Fasano, Schena Editore, 1997, pp. 205-216. Sullo sviluppo dell'«ossessione turca» nel corso del secolo, vedi F. Lestringant, Guillaume Postel et l'"obsession turque", in Guillaume Postel 1581-1981 (Actes du Colloque International d'Avranches 5-9 septembre 1981), Paris, Guy Trédaniel, Éditions de
La Maisnie, 1985, pp. 265-298.

(17) Su questi aspetti, vedi C. VIVANTI, Lotta politica e pace religiosa in Francia fra Cinque e Seicento, Torino, Einaudi, $1974[1963]^{1}$, p. 74 e sg.

(18) Altrove Naselli alleggerisce il testo italiano della tautologia propria dell'originale: «du roy qui regne en Polongne» (476) $\rightarrow$ «del Re di Pollonia» (XXXV, 63). Per una maggiore verosimiglianza, il traduttore corregge il testo francese quando si tratta di descrivere la presa di una città: «emporter en moins de trois heures quatre ou cinq des meilleures villes qui sont sur le Danube» (488) $\rightarrow$ «ottenere in meno di tre mesi, quattro, o cinque delle migliori terre» (XLV, 82-83). 
l'ultimo degli Iagelloni, la corona polacca viene offerta prima a Enrico d'Anjou, che però torna precipitosamente in Francia non appena apprende della sua successione al trono con il nome di Enrico III, quindi a Stefano Bathory, principe di Transilvania. Ma quando Naselli pubblica il Discorso queste considerazioni politiche hanno perso di attualità, tenuto conto che la gravità della situazione è venuta meno con l'elezione a re di Polonia nel 1587 di Sigismondo, nipote di Gustavo Vasa, convertitosi al cattolicesimo.

Lo stesso principio di attualizzazione spiega, crediamo, due ulteriori difformità della resa italiana dal francese.

«Aujourd'huy il semble qu'il y ait peu de differens entr'eux, veu que Monseigneur le duc d'Anjou est depuis peu de jours allé de vie à trespas (qui contestoit contre le Roy Catholique) ne lui estant resté de toutes ses peines que la seule ville de Cambray. On dira qu'elle pourroit bien estre la cause d'amener discorde entre les deux rois d'Espagne et de France; ce que toutesfois je ne pense pas, car ils ne seroient pas si mal-avisez, pour un si petit prix, de jetter leurs royaumes en despense, calamité et ruine» (451).

Si tratta qui di un riferimento a Francesco, duca di Alençon, poi duca d'Anjou dopo la successione al trono del fratello Enrico III. Nato nel 1554, il duca, che conquista Cambrai nel 1581, muore il 10 giugno 1584. Come informa Sutcliffe nelle note di edizione, questo rinvio permette di datare la stesura del presente discorso, scritto probabilmente nell'estate del 1584. Ma Naselli, animato dalla preoccupazione di rendere il messaggio quanto più possibile attuale e privo di personalizzazioni, omette l'intero passo (XIV, 23). Ne consegue un'ulteriore diminuzione della componente soggettiva, processo di appiattimento che ancora una volta colpisce quella sottile complicità che La Noue, dando voce al proprio pensiero, stringeva con il lettore.

Si situano nel medesimo ambito i tagli apportati al capitolo XVI, «Della necessità della pace ne' paesi bassi, et come Sua Maestà Catholica deve concederla»:

«Sa Majesté Catholique, qui est doüee (ce dit-on) de grande debonnaireté, E en fait journellement des preuves en plusieurs, fors qu'en moy, devroit regarder attentivement à cecy, car c'est tout à ses despens que ceste sanglante tragedie se jouë. Maintenant il n'est plus question de l'Estat, la querelle est simplement pour la religion, duquel affaire, encor qu'on ne m'en demande conseil, \& quoy qu'on ne m'en croye, si ne laisseray-je d'en dire un petit mot. Selon mon avis il y faut proceder doucement, ne juger par prejugez, accomoder les loix à la nature du peuple, \& n'adherer pas tant aux rapports de quelques-uns, que aux justes doleances de plusieurs subjets, estant certain qu'il convient avoir de merveilleuses forces, pour forcer les consciences. En fin les choses passees ont demonstré que les princes, qui par guerres ont voulu accompagner la vehemence des prestres, ont defiguré leurs Estat, \& diminué leur grandeur. Et quel Jesuite se pourroit trouver, tant habile fust-il, qui peust persuader à ceux qui ne sont pas Jesuites, que Dieu prend plaisir à tant de sang que les uns \& les autres respandent? Le peuple des Païs-Bas est d'une nature franche; par douceur E bumanité non feinte on remuë les affections de son coeur, mais par coups E injures on l'estrange $\mathcal{E}$ on l'anime. Le plus seur conseil en cecy seroit d'accorder à ceux qui sont armez, la permission qu'ils demandent d'obeïr à Dieu, à fin qu'ils rendissent l'obeïssance que requierent les hommes, car il y a danger que si on ne le fait volontairement, le temps le fera faire par force, lequel peut aussi trop tost produire des accidens favorables pour ceux qui perdent, qu'il a fait pour ceux qui gaignent. Le plus vif aiguillon pour poindre \& inciter les Espagnols à la paix, est de les faire ressouvenir de l'imprudence des François, \& dire, Bella escapata» (452-453) $\rightarrow$ «La Maestà Catholica, che è dolce, et di grande benignità, dovrebbe attentivamente mirare a ciò: perché è a tutte sue spese, che questa sanguinolente tragedia si fa. I popoli del paese basso sono di natura franca, con dolcezza, et humanità non finta, si rimuovono l'affettioni del cuore loro; ma per colpi, et ingiurie, s'allontanano, et inanimano» (XVI, 26). 
Il capitolo risulta notevolmente amputato e le ragioni del rimaneggiamento sono quelle già messe in luce: si escludono i passi in cui è più accentuato l'individualismo dell'autore; non se ne menzionano i rischiosi quanto personali giudizi, in questo caso quelli riguardanti l'operato dei gesuiti ${ }^{19}$; non ci si interroga su problemi che il corso della storia ha risolto (la prospettiva di una pace tra la Francia e la Spagna, che ponga fine alle lotte politiche e religiose, di cui i Paesi Bassi sono da tempo diventati un punto focale, e che è presentata come la necessaria premessa alla guerra contro i turchi, è una questione ancora aperta al momento della stesura dell'originale, ma si avvia verso una possibile soluzione con l'alleanza franco-spagnola conclusasi il 31 dicembre 1584 fra Enrico, duca di Guisa, e Filippo II). Del Discours, che non è mai solo il trasparente resoconto di un evento, Naselli salva in sostanza solo quegli elementi meno evocativi.

La duplice e conciliativa strategia di attualizzazione e di attenuazione, che conferisce al Discorso una garanzia di ricezione in area italiana, si riscontra nella certo non neutrale censura che colpisce la religione protestante. Evidentemente le potenti forze della cristianità non rassicurano del tutto il progetto di crociata, se La Noue scrive dell'innegabile mancanza dei grandi uomini avuti nel passato,

«comme un duc d'Albe, un duc de Guise, le connestable de France, l'admiral de Chastillon, et André Doria pour la mer» (458-459) $\rightarrow$ «come un Duca d'Alva, un Duca di Guisa, il già Connestabile di Francia, et altri; et Andrea Doria per mare» (XXI, 34).

Nella resa italiana si conservano solo i nomi dei difensori della Chiesa cattolica: Andrea Doria, il duca di Montmorency, il duca di Guisa e, a proposito dei Paesi Bassi appena menzionati, il duca d'Alba. Viene escluso significativamente l'unico protestante, Gaspard di Châtillon, meglio noto come l'ammiraglio di Coligny, membro di una delle più importanti famiglie convertite di Francia e vittima del bagno di sangue della notte di San Bartolomeo. Analogo criterio motiva l'esclusione del re di Navarra nel capitolo «De' Capi, che comandassero nell'essercito, et armata Christiana»:

«Pour le regard du corps des François, et de ce qui y seroit adjoint, il se trouveroit assez de princes pour y commander. J'en nommeray seulement deux qui y seroient propres, à sçavoir le roy de Navarre, qui en desir de bien faire et grandeur de courage ne cede à aucun, et monsieur le duc de Lorraine» (477) $\rightarrow$ «per rispetto del corpo de' Francesi, et quanto ci fosse aggiunto, si troverebbono Prencipi assai per comandarci, oltre il Signor Duca di Lorena» (XXXVI, 64).

Perfino i riferimenti ai contrasti tra i cristiani vengono attenuati. Il testo di $\mathrm{Na}$ selli conserva il piano divino da realizzarsi, la messa in scritto della salvezza nel Cristo, ma omette quelle parole che, facendo appello alla quintessenza dell'insegnamento evangelico «et si regnum in se dividatur non potest stare regnum illud / et si domus super semet ipsam dispertiatur / non poterit domus illa stare» $(M c, 3,24-25)$, rischierebbero di dar voce alle reali scissioni interne alla Chiesa:

«je sçay bien qu'il y a entre nous des disputes sur le fait de la religion. Pour cela les catholiques et evangeliques ne laissent pas d'estre freres, entez sur un mesme tronc, qui est Jesus Christ» (515-516) $\rightarrow$ «Io so bene, che tra noi Christiani ci sono alcune dispute. Non perciò resta, che tutti non siamo fratelli inseriti in un medesimo tronco, ch'è GIESU CHRISTO» (LVIII, 127).

(19) Ricordiamo che i Discours furono duramente attaccati dal gesuita Antonio Possevino in Judicium de Nuae militis Galli. Joannis Bodini, Philippi Mornaei et Nicolai Machiavelli quibusdam scriptis, Lugduni, 1593, in- $8^{\circ}$ (testo conservato alla Bibliothèque Nationale de France, insieme ad un'edizione del 1693 ed una del 1694). 
Nel contesto di questi interventi sono anche da ricondurre alcune modifiche, di aspetto linguistico e stilistico, utili per aiutarci a cogliere le modalità di traduzione ricorrenti nel lavoro di Naselli e intimamente legate alla cultura del Cinquecento. Il carattere anti-italiano dei Discours - evidenziato giustamente da Anna Bettoni ${ }^{20}$-, pur apparendo meno evidente in questo Discorso, ci sembra ad esempio motivare due casi di inversione delle parole, quando Naselli decide di menzionare prima il popolo italiano, quindi quello spagnolo: «la nation espagnolle et italienne» $(490) \rightarrow$ «natione Italiana, et Spagnuola» (XLVI, 85); «quatre mille Espagnols, et autant d'Italiens» (510) $\rightarrow$ «quattro mila Italiani, et altretanti Spagnuoli» $(\mathrm{LV}, 117)$. Il risultato implicito allo spostamento dei sostantivi all'interno della frase non è, per il lettore, solo un dettaglio stilistico, ma una scelta riconducibile a chiare motivazioni di strategia editoriale, volte a dare maggior risalto alla posizione dell'Italia nella crociata $^{21}$. Dovendo decidere della soluzione di problemi atti a semplificare e scomporre il messaggio, anche per il Discorso di La Noue il traduttore applica criteri sostanzialmente simili a quelli cui è già ricorso per i testi di Lucinge e di Montaigne. Per porre l'accento sull'andamento concreto e piano della rappresentazione - segno, si è detto, di elementare realismo e fiuto per gli affari dell'editore -, Naselli diminuisce l'intensità stilistico-figurativa delle immagini (magari variando anche solo uno degli elementi che le compongono), il cui carattere di prova si regge ora a discapito soprattutto di figure retoriche come la metafora o la metonimia:

«Quelqu'un adjoustera, que les petits potentats sont bien aises que les grands s'entremangent. Ils auroyent tres-bonne raison si les grands les vouloient devorer» (451) $\rightarrow$ «alcuno aggiungerà, che i piccioli Potentati hanno a caro, che i grandi tra loro si distruggano. Essi havrebbono buona ragione, se i grandi volessero devorarli» (XIV, 24);

«C'est chose deplorable de voir ceux qui adorent un mesme Christ s'entre-poursuyvre à feu \& à sang, comme bestes sauvages» $(452) \rightarrow$ «Cosa pietosa è di vedere quelli, che adorano un'istesso Christo, farsi guerra a fuoco, et a sangue» (XIV, 25);

«Leur harquebuserie, qui se seroit attaquee avec la nostre porteroit alors la peine de sa temerité» $(487) \rightarrow$ «Gli archibugieri, che si fossero attaccati con i nostri, porterebbono all'hora la pena della loro temerità» (XLIV, 80);

«ceste mortelle et sanglante tragedie se trouveroit embellie par la diversité de tant de nouveaux actes. Mais celuy que je veux reciter» (509) $\rightarrow$ «questa mortale, et sanguinolente Tragedia si ritroverebbe ornata con la diversità di tante nuove attioni. Ma quello ch'io voglio hora ricordare» (LV, 117);

«leurs corps sanglans estendus morts dans les ruës» $(513) \rightarrow$ «i corpi loro sanguinolenti distesi a terra» $(\mathrm{LV}, 122)$;

(20) Art. cit., p. 169. Bettoni si sofferma sul problema grafico del termine francese «Archipelago» [«l'Archipelago» (489) $\rightarrow$ «l'Arcipelago» (XLVI, 84); «Archipelago» (498) $\rightarrow$ Arcipelago (L, 98); vogliamo segnalare anche un'omissione nella resa italiana: «l'armee pourroit encor nettoyer de Turcs quelques isles de l'Archipelago» (495) $\rightarrow$ «l'Armata potrebbe liberare dalle mani de'Turchi alcune isole» (XLVIII, 92)], osservando che «pur nella sua qualità minima di dettaglio linguistico e quasi solo ortografico, la parola 'Archipelago' ci permette di riconoscere nel testo dei Discours la presenza del lettore di Bordone e di altri portolani italiani» (p. 169). Su -archi, arci, vedi anche F. LESTRINGANT, L'Archipel comme mer négative: la mer Égée dans les "Isolarii" de la Renaissance, in L'Europa e il Levante nel Cinquecento. II, op. cit., pp. 5-31.

(21) In «QUELLES VOYES ET PRO / cedures sont plus propres pour user / au redressement de l'Estat. QUATRIEME DISCOURS» La Noue sostiene che, per salvare la Francia, sia meglio ricorrere alla «douceur», alla «concorde» e ai «bons exemples de vie» piuttosto che alle armi, come invece vorrebbero $\mathrm{i}$ «passionez Catholiques». Il già ricordato ambiguo dualismo di certe posizioni di La Noue si ritrova in alcuni passi riguardanti i contrasti tra gli ugonotti e i papisti da un lato e i francesi e gli italiani dall'altro. Sempre più presenti in Francia, gli italiani sono oggetto di critiche e atteggiamenti negativi da parte dei francesi, ma ecco che La Noue, inaspettatamente, presenta argomenti a loro difesa (104 e sg.). 
«tenter un party d'bommes reduits au dernier desespoir» (511) $\rightarrow$ «tentare l'ultima disperatione» $(\mathrm{LV}, 120)^{22}$.

A prescindere dai diversi orientamenti ideologici dei tre autori, si riscontra da parte del traduttore un'analoga volontà di chiarificazione. Per rimanere in ambito semantico, alcuni tratti ricorrono in modo costante, benché diversamente distribuiti, nella resa italiana delle opere francesi. Nel Discorso, alle ricordate sinonimie glossanti si aggiunge così l'uso di sinonimi e di variazioni volto a destituire il testo originale di ogni funzione espressiva o suasoria, per aumentarne invece il rapporto col reale: «abondance de munitions» (500) $\rightarrow$ «munitioni in abbondanza» (LI, 101); «un beau pourtrait» (441) $\rightarrow$ «un bello esempio» (IV, 6); «ses pere \& grand pere d'heureuse memoire ont establis» (448) $\rightarrow$ «che i suoi antecessori hanno stabiliti» (XI, 18); «par la poincture de tant d'aiguillons» (454) $\rightarrow$ «punti da tanti stimoli» (XVII, 28); «ce langage» (491) $\rightarrow$ «questa maniera di parlare» (XLVII, 86); «l'armee imperiale se devroit placer» (494) $\rightarrow$ «l'essercito imperiale dovrebbe pigliare piazza» (XLVIII, $92) ;$ «departir le gasteau» $(514) \rightarrow$ «partire la preda» (LVII, 125) ${ }^{23}$. Né si può tacere che le perdite tendenti a semplificare il testo originale possono indurre a degli errori. È l'esempio macroscopico di alcuni stati europei che subiscono un cambiamento di natura geografica, omissione forse imputabile ad una lettura errata dei segni d'interpunzione presenti nella costruzione della frase francese: «le demourant sont isles, comme Angleterre, Escosse, Dannemarc \& Suede, qui sont comme peninsules» (445) $\rightarrow$ «Il resto sono isole, come Inghilterra, Scotia, Danimarca, \& la Svevia» (IX, 14).

Ad alterare la fedeltà del testo di arrivo contribuiscono inoltre alcuni scarti relativi ai numeri, divergenze che nel Discorso non sembrano rientrare tra gli errori paleografici, ma sembrano piuttosto legati ad una volontà di semplificazione (specie nei casi di numerazione su base venti - in parte continuati anche nel francese attuale, ma totalmente estranei all'italiano -, e nel ricorso alla formula «et, o più») da parte del traduttore. Eccone gli esempi:

«un nombre de cinq ou six mille petits enfants qu'ils arrachent tous les ans du sein de leurs meres plorantes» (441-442) $\rightarrow$ «un numero di cinque, o se mila fanciulli, et più, ch'essi tirano ogn'anno dal petto delle loro madri lagrimanti» (VI, 8); «cent ou six vingt ans» (467) $\rightarrow$ «cento sessant'anni» (XXIX, 49); «avec cent ou six vingts mille» (475) $\rightarrow$ «con centomila, o più» (XXXIV, 61); «avecques pertes de plus de quatre ou cinq mille chevaux» (487) $\rightarrow$ «con perdita di più di cinque mila cavalli» (XLIV, 80);

(22) Alla semplificazione del messaggio contribuiscono inoltre alcuni cambiamenti che, pur di minore importanza, è bene comunque segnalare. Essi riguardano l'omissione dei determinanti: «Des pauvres chrestiens orientaux» $(452) \rightarrow$ «de' poveri Christiani» (XIV, 25); «une sotte prophetie» (471) $\rightarrow$ «una Profetia» (XXXII, 54); «les autres vaisseaux ronds» (475) $\rightarrow$ «gl'altri vascelli» (XXXV, $62)$; «vostre petite guerre» (502) $\rightarrow$ «la vostra guerra» (LII, 105); «bien combatre» $(512) \rightarrow$ «combattere» (LV, 121); «ayans bien preveu» $(511) \rightarrow$ «havendo previsto» (LV, 119); e i cambiamenti relativi all'uso della negazione: «à moy qui n'ay jamais veu» $(437) \rightarrow$ «a me, che poco ho veduto» (1); «je ne diray rien» $(450) \rightarrow$ «io non dirò altro» (XIII, 22); «de nul fruit» $(450) \rightarrow$ «di poco frutto» (XIV, 23).

(23) Nella resa dei sostantivi segnaliamo anche il cambiamento dalla forma singolare a quella plura- le: «si nous ne nous aidons des remedes que Dieu par sa bonté nous met en la main» (444) $\rightarrow$ «se noi non ci aiutiamo de'rimedij, che Dio per bontà sua ci pone nelle mani» (VIII, 13); «autre chose pareille» (513) $\rightarrow$ «altre cose simili» (LV, 123); «les Turcs, qui resteroient, iroient se jeter dans les fossez de leur retranchement, tout semé d'artillerie et d'barquebuserie» (510) $\rightarrow$ «i Turchi, che restassero, si lancierebbono dentro i fossi de' loro ritrinciamenti, seminati tutti, et forniti di artiglierie, et archibugerie» (LV, 118-119); nonché il passaggio inverso: «ils esprouveront ses vengeances sans misericorde» (471) $\rightarrow$ «proveranno la sua vendetta» (XXXI, 53, con omissione); «avecques pieds de plomb et mains de fer» $(491) \rightarrow$ «co'l piede del piombo, et mani di ferro» (XLVII, 86); «les maladies» (501) $\rightarrow$ «l'infirmità» (LI, 103); «les courages ne sont pas diminuez» $(457-458) \rightarrow$ «l'ardire non è diminuito» (XXI, 34). 
«ceux qui depuis deux cens ans» (489) $\rightarrow$ «quelli, che doppo cent'anni» (XLV, 83); «deux cens vingt mille hommes» (502) $\rightarrow$ «ducento mila, et più huomini» (LI, 104); «six vingts galeres» $(511) \rightarrow$ «ben cento, o più galere» (LV, 120); «cens ou six vingts galeres» $(514) \rightarrow$ «cento venti galere» (LVI, 124); «tiendroient leur armee à six lieuës pres logee fortement, pour les favoriser» (493) $\rightarrow$ «tenendo l'essercito loro distante cinque, o sei leghe solamente in alloggiamento forte per favorirli» (XLVIII, 90).

Anche in ambito verbale le modifiche apportate da Naselli sembrano obbedire ai criteri verificati nella resa italiana delle altre due traduzioni. Si riscontrano infatti, pur se meno frequenti, alcuni cambiamenti nella resa dei tempi verbali: «laquelle leur a cousté tres-cher» (439) $\rightarrow$ «la quale costa loro molto cara» (4); «qui fut onques» (441) $\rightarrow$ «che mai sia stata» $(\mathrm{V}, 7)$; due passaggi dalla forma verbale a quella sostantivale: «le desir d'entreprendre» (473) $\rightarrow$ «il desiderio dell'impresa» (XXXIV, 59); «le premier rang d'assaillir» (509) $\rightarrow$ «il primo luogo all'assalto» (LV, 117); il caso inverso, di poco più presente: «les combats» (495) $\rightarrow$ «il combattere» (XLIX, 94); «ce qui les faict autant penser à la fuite qu'à combattre» $(498) \rightarrow$ «il che li fa pensare altretanto al fuggirsene, che al combattere» (L, 98); «ils prennent baleine \& se preparent» $(440) \rightarrow$ «essi respirano, et si preparano» (4); «pour cercher [sic] les cachettes» (512) $\rightarrow$ «per cercare d'ascondersi» (LV, 121-122); e infine un esempio di riduzione verbale, legato al concetto di disciplina (militare), che i soldati devono «garder et observer» (460) $\rightarrow$ «osservare» (XXII, 35).

Il tentativo di individuare le ragioni delle modalità di traduzione di Girolamo Naselli ci ha condotto, specie con gli ultimi rilievi, dalla resa della linea e delle idee del testo di La Noue a un piano tecnico-linguistico che ci permette di inserire il $\mathrm{Di}$ scorso in un più vasto contesto di fortunate traduzioni a cavallo tra Cinque e Seicento. In questa prospettiva, è chiaro che il valore ideologico-letterario dell'originale - nel quale l'autore parla in prima persona, avviandosi ad un dialogo con il lettore, e sottolinea i punti di unione fra cattolici e riformati - trova un accentuato interesse anche per lo storico della lingua, che riconosce, nelle pagine del testo di arrivo, la preoccupazione di mantenere la funzione informativa-dottrinale della parola ancorandola tuttavia al nuovo contesto storico-sociale cui il testo si inserisce. Le oggettive difficoltà di resa linguistica presentatesi al traduttore, con il loro carattere spesso essenzialmente storico, sono tutti motivi comuni a fondamento di una tradizione nella quale ogni evento isolato contribuisce all'affermazione di un ampio sistema di ricezione, nel caso specifico quello, cui ci siamo più volte riferiti, degli orizzonti d'attesa del pubblico italiano.

La vastità dei problemi sollevati dall'opera di La Noue - dalle invocazioni al «Seigneur des batailles» (Discours I, 31), per porre fine alle discordie da cui era lacerata la Chiesa, alla forza di certe ideologie a lui coeve - si concentra nel Discorso ferrarese sulle «cose turchesche», una situazione specifica che, conforme ai progetti di crociata dei Duchi di Ferrara, di Mantova e del Papa, costituisce da anni un filone di preferenza nel catalogo baldiniano ${ }^{24}$. Questo interesse spiega la scelta del trattato, nel quale il vigile senso critico dell'editore individua quei punti cui va il suo interesse preminente, ispirato da considerazioni di convenienza che ne consentano l'accesso ad una vasta cerchia di utenti. A questo fine il traduttore opera la resa italiana, secondo alcuni principi di fondo non dissimili da quelli cui aveva obbedito per il «volgarizzamento» degli Essais di Montaigne, «in un triplice processo di generalizzazione, di 
appiattimento, di esplicitazione che - osserva Anna Maria Raugei - sfalda e disgrega, a livello linguistico e stilistico, la densità, il vigore, la forza espressiva dell'originale ${ }^{25}$. Tuttavia in questa traduzione dell'opera di La Noue, Naselli è certo favorito dal carattere forse più «pratico» del testo, nel quale l'autore, «uomo universale» sotto varî aspetti della vita, ma dalla scienza evidentemente molto meno libresca di quella di Montaigne, vede il Male e ne indica i possibili rimedi. E infatti, il carattere di propaganda del Discorso sembra favorire il progetto di difesa della Cristianità che le corti italiane, spaventate dalla ripresa dell'avanzata turca verso l'Europa centrale o verso l'Italia, stavano sollecitando con inviti diretti al Cristianissimo affinché prendesse parte alla Crociata. Come aveva osservato lo scrittore veneziano Lazaro Soranzo nel suo fortunato Ottomanno (edito a Ferrara nel 1598, 1599 e 1607), la guerra contro il turco è il «maggior negotio, ch'ora corra nel Mondo». In tali circostanze, il testo di La Noue acquista davvero una grande importanza, e le intenzioni del traduttore determinano un materiale che avrà vita propria, sostenuto dalla nuova comunità che se ne servirà.

RICCARDO BENEDETTINI 